EXTENDED REPORT

\title{
Classification of abnormal fundus autofluorescence patterns in the junctional zone of geographic atrophy in patients with age related macular degeneration
}

\author{
A Bindewald, S Schmitz-Valckenberg, J J Jorzik, J Dolar-Szczasny, H Sieber, C Keilhaver, \\ A W A Weinberger, S Dithmar, D Pauleikhoff, U Mansmann, S Wolf, F G Holz, for the FAM \\ Study Group*
}

Br J Ophthalmol 2005;89:874-878. doi: 10.1136/bjo.2004.057794

See end of article for authors' affiliations

Correspondence to: Professor Frank G Holz, Department of Ophthalmology, University of Bonn, Ernst-Abbe-

Strasse 2, D-53127 Bonn Germany; frank.holz@

ukb.uni-bonn.de

*Fundus Autofluorescence in Age-related Macular Degeneration Group. See appendix for list of participants.

Accepted for publication 2 December 2004

\begin{abstract}
Aim: To describe and classify patterns of abnormal fundus autofluorescence (FAF) in the junctional zone of geographic atrophy (GA) in patients with age related macular degeneration.

Methods: Digital FAF images were recorded in 164 eyes of 107 patients using a confocal scanning laser ophthalmoscope (cSLO; excitation $488 \mathrm{~nm}$, detection above $500 \mathrm{~nm}$ ) as part of a prospective multicentre natural history study (FAM Study). FAF images were obtained in accordance with a standardised protocol for digital image acquisition and generation of mean images after automated alignment.

Results: Image quality was sufficient for classification of FAF patterns in 149 eyes $(90.9 \%)$ with lens opacities being the most common reason for insufficient image quality. Abnormal FAF outside GA in 149 eyes was classified into four patterns: focal $(12.1 \%)$, banded (12.8\%), patchy $(2.0 \%)$, and diffuse $(57.0 \%)$, whereby $12.1 \%$ had normal background FAF in the junctional zone. In $4 \%$ there was no predominant pattern. The diffuse pattern was subdivided into four groups including reticular (4.7\%), branching (27.5\%), fine granular (18.1\%), and fine granular with peripheral punctate spots $(6.7 \%)$.

Conclusions: Different phenotypic patterns of abnormal FAF in the junctional zone of GA can be identified with cSLO FAF imaging. These distinct patterns may reflect heterogeneity at a cellular and molecular level in contrast with a non-specific ageing process. A refined phenotypic classification may be helpful to identify prognostic determinants for the spread of atrophy and visual loss, for identification of genetic risk factors as well as for the design of future interventional trials.
\end{abstract}

n developed countries age related macular degeneration (AMD) has become the most common cause for registered blindness. Severe visual loss results from choroidal neovascularisation (CNV), pigment epithelial detachment or geographic atrophy (GA) of the retinal pigment epithelium (RPE). ${ }^{1}$ GA is thought to be the natural end stage of the atrophic AMD process when CNV does not develop. ${ }^{2}$ While $\mathrm{CNV}$ is the more common cause for visual loss, GA is responsible for approximately $20 \%$ of the severe visual impairment from AMD. ${ }^{3}$ While extensive research in recent years has been attributed to the pathomechanisms of CNV formation and its inhibition, relatively little attention has been focused on GA as a common disorder.

With the advent of confocal scanning laser ophthalmoscopy (CSLO) it has become possible to visualise fundus autofluorescence (FAF) and its spatial distribution in vivo. ${ }^{5-8}$ It represents a tool to evaluate the RPE during ageing and in retinal disease. ${ }^{9}$ As shown by spectrometric investigations by Delori and associates FAF is mainly derived from lipofuscin (LF) in the RPE. ${ }^{10}$ Excessive accumulation of LF represents a common pathogenetic pathway in various monogenetic and complex retinal diseases and may precede photoreceptor degeneration. ${ }^{11-13}$ Recent studies described FAF changes in early and advanced atrophic AMD. ${ }^{7}{ }^{14}$ Hereby, excessive LF accumulation in the junctional zone precedes the development of GA and the enlargement of pre-existing GA. ${ }^{15}$ Preliminary observations using FAF imaging suggested that there may be distinct phenotypes with regard to changes in FAF in the junctional zone of GA. ${ }^{7}$ Based on these findings a prospective, multicentre, natural history study was initiated to more precisely identify FAF variations in patients with
AMD. Here we report on a refined classification of FAF patterns in the junctional zone of GA in patients with AMD.

\section{METHODS}

Digital FAF images were obtained as part of the prospective, multicentre Fundus Autofluorescence in Age-related Macular Degeneration (FAM) Study using a cSLO (Heidelberg retina angiograph, HRA classic, and HRA 2, Heidelberg Engineering, Dossenheim, Germany), the optical and technical principles of which have been described previously. ${ }^{75}$

For FAF imaging, an argon blue laser (HRA classic) or an optically pumped solid state laser (HRA2) (both $488 \mathrm{~nm}$ ) was used for excitation. Emitted light was detected above $500 \mathrm{~nm}$ (barrier filter). With an interference filter, the green share of the argon laser light was reduced to the extent that the ratio of the intensities of green and blue is below $10^{-7}$. The above mentioned cut-off filter suppressed blue argon excitation light at $488 \mathrm{~nm}$ by a factor of $10^{-6}$. Consequently, it was assumed that reflectance signals do not contribute to the FAF image obtained from the posterior pole of the examined eye. This blockage even suppressed reflexes on the optical surface of the lens.

Maximal retinal irradiance using the HRA was approximately $2 \mathrm{~mW} / \mathrm{cm}^{2}$ for a $10^{\circ} \times 10^{\circ}$ frame and was therefore well below the limits established by the American National

\footnotetext{
Abbreviations: AMD, age related macular degeneration; cSLO, confocal scanning laser ophthalmoscope; ETDRS, Early Treatment Diabetic Retinopathy Study; FAF, fundus autofluorescence; GA, geographic atrophy; HRA, Heidelberg retina angiograph; LF, lipofuscin; $\mathrm{RPE}$, retinal pigment epithelium; VA, visual acuity
} 


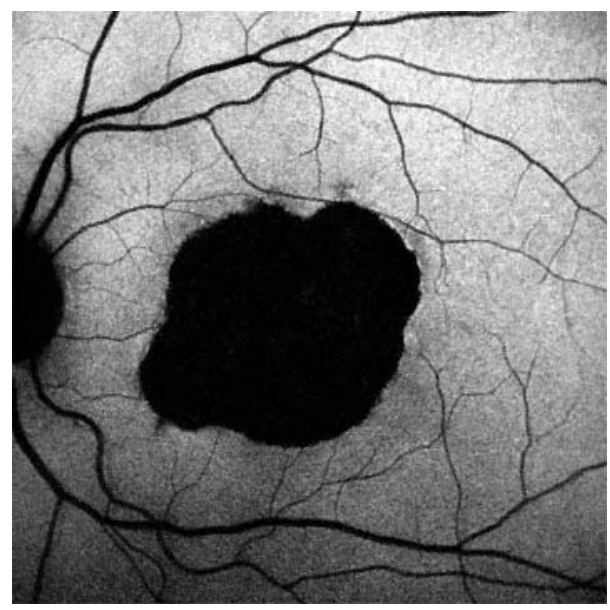

Figure 1 Normal background fundus autofluorescence outside the central patch of geographic atrophy (66 year old patient, left eye, VA $5 / 80$ ). The atrophic area is associated with a markedly decreased fundus autofluorescence because of the absence of retinal pigment epithelial cells, and, thus, autofluorescent lipofuscin.

Standards Institute (ANSI) and other international standards (ANSI Z136.1; 1993). Images were immediately digitised and processed using a flexible frame processor and subsequently displayed on a computer screen. The digital images were saved on hard disc for further analysis and processing.

For acquisition of FAF images a standard operation procedure was developed which included focusing of the retinal image in reflection and red-free mode, sensitivity adjustment and acquisition of at least 15 single $30^{\circ} \times 30^{\circ} \mathrm{FAF}$ images which encompassed the entire macular area and at least part of the optic disc. In order to amplify the FAF signal the best nine single images were aligned and a mean image was calculated after detection and correction of eye movements using image analysis software (Heidelberg Eye Explorer, Heidelberg Engineering, Dossenheim, Germany).

Patients with unilateral or bilateral GA caused by AMD were included into the study. Any sign of exudation or choroidal neovascularisation as well as previous drug, surgical, or laser therapy were exclusion criteria. The study followed the tenets of the Declaration of Helsinki and was approved by the local ethics committees. Informed consent was obtained from each patient after explanation of the nature and possible consequences of the study. Best corrected central visual acuity (VA) was determined using Early Treatment Diabetic Retinopathy Study (ETDRS) charts. EDTA blood samples were taken for future molecular genetic analyses. The pupil of the study eye was dilated with $1 \%$ tropicamide and FAF images were obtained from each patient.

FAF images were evaluated by two independent readers. In case of discrepancy a third reader was involved to arbitrate. Abnormal FAF was defined as an increased or decreased FAF signal compared with the FAF outside such lesions, the latter being referred to as normal FAF, which shows an even distribution, with a typical lower intensity in the macula caused by absorption of macular pigment in the neurosensory retina and lower LF levels in central RPE cells. ${ }^{56}$ There is also a lower signal along large retinal vessels (absorption) and at the optic disc (absence of autofluorescent material).

\section{RESULTS}

Digital FAF images were obtained from a total of 164 eyes from 107 patients (mean age 72.96 (SD 7.43) years; 44 male and 63 female patients) with unilateral or bilateral GA caused by AMD.
Table 1 Variations in fundus autofluorescence (FAF) in the junctional zone of geographic atrophy (GA) of 149 eyes* with atrophic AMD

\begin{tabular}{lcr}
\hline $\begin{array}{l}\text { Pattern of increased FAF in the } \\
\text { junctional zone of GA }\end{array}$ & Not & $\%$ \\
\hline None & 18 & 12.1 \\
Focal & 18 & 12.1 \\
Banded & 19 & 12.8 \\
Patchy & 3 & 2.0 \\
Diffuse & 85 & 57.0 \\
$\quad$ Reticular & 7 & 4.7 \\
$\quad$ Branching & 41 & 27.5 \\
$\quad$ Fine granular & 27 & 18.1 \\
$\quad$ Fine granular with peripheral & 10 & 6.7 \\
$\quad$ punctate & & \\
\hline
\end{tabular}

*In 15 eyes of the initially included 164 eyes insufficient FAF image quality did not allow for evaluation of the FAF pattern outside the atrophic patch; frequency data therefore refer to 149 eyes.

†Six eyes $(4.0 \%)$ were not grouped into one of these types, because there was no predominant FAF pattern in the junctional zone of GA.

Sixty eyes (36.6\%) had multifocal areas of atrophy whereas 103 eyes $(62.8 \%)$ presented with an unifocal patch of GA. In one eye $(0.6 \%)$ delineation of the GA was not clear and the distinction between unifocal and multiple confluent areas of atrophy therefore impossible. An extrafoveolar location of the atrophic areas was observed in 86 eyes $(52.4 \%)$; GA involved the foveola in 77 eyes $(47.0 \%)$. In one eye $(0.6 \%)$ the atrophic patch seemed to reach the fovea, but an exact demarcation was not possible. Because VA was 10/25 the foveola probably was not involved in the atrophic process. The total area of atrophy ranged from $0.4-39.8 \mathrm{~mm}^{2}$ (mean $6.6 \mathrm{~mm}^{2}$ ). Mean VA in eyes with extrafoveolar GA was 10/20 (range 5/200-20/ 16 ), and $10 / 50$ (range 5/200-20/20) in eyes with subfoveal GA.

GA as well as retinal vessels and the optic disc typically showed a markedly decreased FAF signal as previously described. ${ }^{15-17}$ Evaluation of the FAF pattern outside GA requires good image quality and was performed in FAF images with sufficient image quality regarding contrast and sharpness. FAF image quality was sufficient for evaluation in $149(90.9 \%)$ out of 164 eyes. Cataract and inability of proper fixation during the image recordings represented the most common reasons for insufficient image quality. Frequency data therefore refer to 149 eyes. In 18 eyes (12.1\%) there was

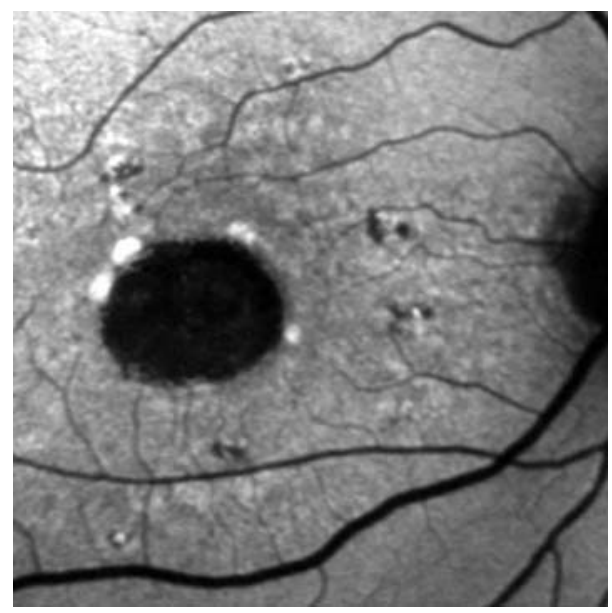

Figure 2 Multiple patches of markedly focal increased fundus autofluorescence at the margin of the atrophic patch (77 year old patient, right eye, VA 5/80). 
no abnormal FAF noted in the junctional zone. The FAF outside the GA (fig 1) showed a normal even distribution.

The following patterns of abnormal FAF in the junctional zone of GA were identified (table 1).

\section{Focal increased autofluorescence}

In 18 eyes ( $12.1 \%)$ single or multiple small spots of markedly focal increased FAF were present at the margin of the atrophic patch (fig 2).

\section{Band of increased autofluorescence}

In 19 eyes $(12.8 \%)$ there was a continuous stippled band of increased FAF surrounding the entire atrophic area (fig 3 ).

\section{Patchy increased autofluorescence}

Three eyes $(2.0 \%)$ showed larger areas of patchy increased FAF outside the area of GA. FAF intensity of these patches tended to be of lesser degree compared with the focal pattern described above (fig 4).

\section{Diffuse increase of autofluorescence}

The most frequent pattern of increased FAF in eyes with GA was a pattern referred to as diffuse, which was noted in 85 eyes $(57.0 \%)$. Hereby, FAF changes were not confined to the margin of the atrophic areas but showed a larger spread at the posterior pole. These diffuse changes showed again interindividual differences that were classified into four subtypes.

\section{Reticular}

A so called reticular pattern (seven eyes, 4.7\%) was characterised by various lines of increased FAF with a preferred radial orientation (fig 5A).

\section{Branching}

In 41 eyes $(27.5 \%)$ there was a diffuse increased FAF with a fine branching pattern of an increased FAF signal (fig 5B).

\section{Fine granular}

This pattern was characterised by a larger area of increased FAF with a granular-like appearance surrounding the GA, and was present in 27 eyes (18.1\%) (fig 5C). There was a clear border between the granular increased FAF and the surrounding normal background FAF.

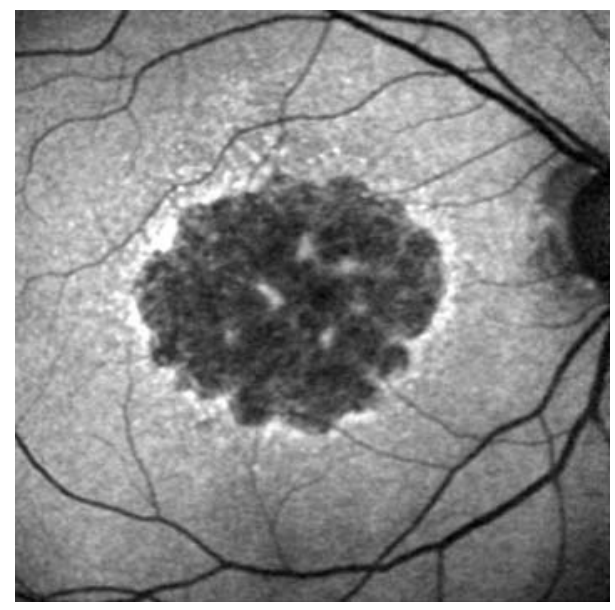

Figure 3 Band of increased fundus autofluorescence surrounding the geographic atrophy (69 year old patient, right eye, VA 10/40).

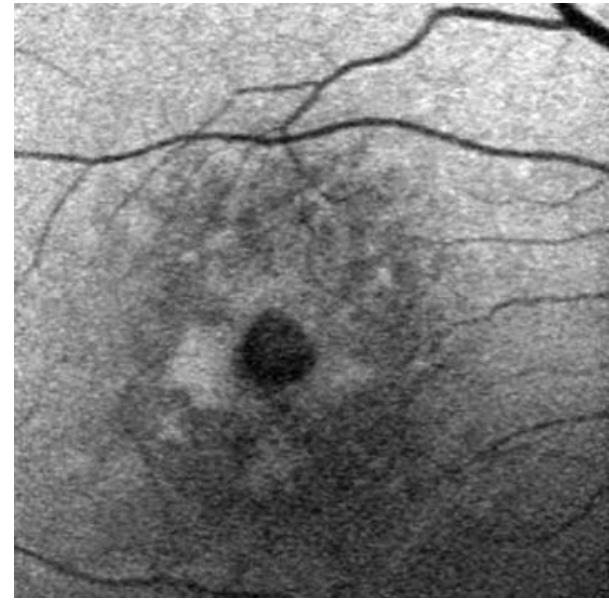

Figure 4 Larger areas of patchy increased fundus autofluorescence outside the area of geographic atrophy $(66$ year old patient, right eye, VA $10 / 20)$

Fine granular with peripheral punctate spots In 10 eyes $(6.7 \%)$ with a diffuse FAF change surrounding the atrophic area there were elongated small lesions with increased FAF signal (fig 5D).

Six eyes $(4.0 \%)$ were not grouped to one of these types. There was no predominant FAF pattern in the junctional zone of GA which led to discrepancy between the different readers. Frequency data of table 1 therefore show the results of 143 eyes of 97 patients. Bilateral GA was present in 46 patients. In 41 patients $(89.13 \%)$ the pattern of abnormal FAF outside the GA was identical reflecting a high degree of intraindividual symmetry.

\section{DISCUSSION}

With the advent of confocal scanning laser ophthalmoscopy it is possible to visualise FAF in vivo. While preliminary reports indicated interindividual variations in association with GA in patients with $\mathrm{AMD}^{7}$ a refined classification of FAF changes in the junctional zone of GA is reported here. Refined phenotyping is not only important to identify prognostic determinants, it appears also as a prerequisite to determine specific genetic factors in a complex, multifactorial disease such as AMD. ${ }^{18}{ }^{19}$ We propose that the FAF classification system presented here may be used in other studies on GA caused by AMD.

RPE LF contains the dominant fluorophores responsible for FAF. ${ }^{20}$ Excessive LF accumulation represents a common final pathogenetic pathway in various monogenetic and complex retinal disorders. LF granules also accumulate with age. ${ }^{21}$ Recent experimental studies have addressed possible molecular mechanisms of how excessive LF may interact with normal cellular functions of RPE cells. Hereby, A2-E has been identified as a main autofluorescent compound of LF. ${ }^{22}$ Toxic properties of A2-E include phototoxic and detergent effects as well as inhibitory effects on lysosomal function. ${ }^{23-26}$ Identification of other molecular species besides the LF proteome is in progress. ${ }^{27}$ Recently developed animal models that share phenotypic characteristics of AMD including excessive LF and A2-E accumulation in the RPE, add to the understanding of potential underlying molecular mechanisms. ${ }^{28}{ }^{29}$ Clinical evidence of adverse LF effects comes from FAF investigations and psychophysical studies. Scholl et al have demonstrated impaired photoreceptor function in association with increased FAF by fine matrix mapping in patients with AMD including GA. ${ }^{30}$ We have shown that increased FAF in the junctional zone of GA and, thus, 

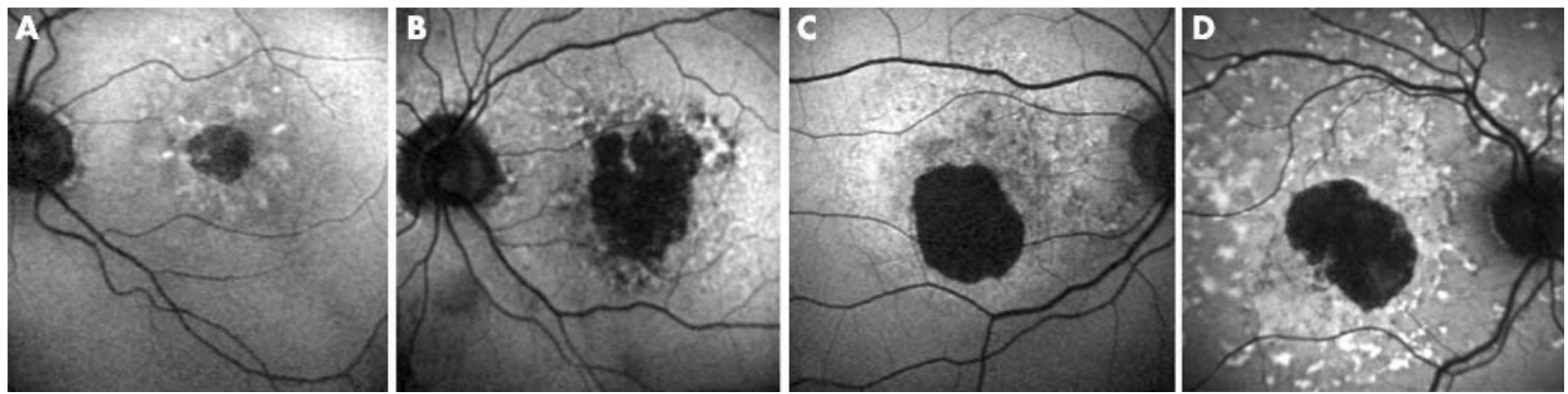

Figure 5 Diffuse types of increased fundus autofluorescence in the junctional zone of geographic atrophy. (A) Reticular pattern of increased fundus autofluorescence with a preferred radial orientation (69 year old patient, left eye, VA 10/32). (B) Diffuse pattern of increased fundus autofluorescence with relatively short branching linear features (79 year old patient, left eye, VA 10/32). (C) Diffusely increased fundus autofluorescence with a granular-like appearance surrounding the well defined area of geographic atrophy (55 year old patient, right eye, VA 10/20). (D) Peripheral elongated small lesions with increased fundus autofluorescence signal (62 year old patient, right eye, VA 10/100).

excessive LF accumulation precede the development of new areas of GA or the enlargement of pre-existing atrophic patches, ${ }^{7}$ and is associated with impaired retinal sensitivity on microperimetry testing. ${ }^{31}$

Various limitations have to be considered for FAF imaging. Media opacities including lens opacifications may result in FAF images that cannot be analysed adequately. Furthermore, this system currently does not allow for absolute quantitation of the FAF signal. However, this is not a prerequisite to delineate topographic patterns of abnormal FAF and, therefore, it might not be required to detect absolute levels of FAF. The accurate delineation of atrophic areas is facilitated in FAF imaging compared to fundus photographs as a result of the absence of the RPE containing the dominant fluorophores. ${ }^{32} 33$

Rückmann et al initially described the presence of elevated FAF surrounding GA. ${ }^{14}$ Different abnormal FAF patterns in the junctional zone of GA in association with AMD have been identified previously in a smaller cohort. ${ }^{7}$ Based on these findings the hypothesis was raised that there may be marked interindividual variation in the presence of high intraindividual symmetry with distinct phenotypic patterns in FAF. ${ }^{33}$ Here we examined patients using FAF imaging. Particular features of FAF changes allowed for classification into various patterns.

GA in association with AMD has been shown to gradually enlarge over time. ${ }^{34-37}$ Schatz and McDonald determined the yearly spread of atrophy in fundus photographs. ${ }^{36}$ Medium sized areas of atrophy grew faster than smaller areas. Sunness et al determined rates of spread in a cohort of 123 patients and identified interindividual variaton. ${ }^{27}$ So far, prognostic determinants for the individual progression of atrophic $\mathrm{AMD}$, and, therefore, additional visual loss, are unknown. Since there is both experimental and clinical evidence of toxic properties of $\mathrm{LF}^{23-26}$ we hypothesise that different phenotypes of FAF changes in the junctional zone of GA are associated with different rates of spread of preexisting atrophy. Our preliminary analyses indicate that progression is smaller in eyes with no or minimal FAF alteration in the junctional zone as opposed to eyes with widespread diffuse changes (Bindewald et al ARVO 2004; \#2960). As different patterns of increased FAF might reflect heterogeneity at a cellular and molecular level, this classification might be helpful to identify genetic factors. The high degree of symmetry of abnormal FAF in patients with bilateral GA points rather towards specific genetic contributions than non-specific ageing processes. ${ }^{33}$

In summary, based on a cross sectional analysis of patients with GA associated with AMD a classification system is presented to distinguish phenotypic patterns of FAF alterations in the junctional zone of atrophic areas. The observations implicate excessive RPE LF accumulation in atrophic AMD and are useful to test hypotheses in this respect. Non-invasive FAF imaging obviously gives information over and above conventional imaging methods. Expanding the ongoing FAM study with longitudinal analyses it may be useful to identify prognostic determinants for the progression of atrophic $\mathrm{AMD}$, to monitor future interventional trials and to determine genetic factors.

\section{Authors' affiliations}

A Bindewald, S Schmitz-Valckenberg, F G Holz, Department of Ophthalmology, University of Bonn, Ernst-Abbe-Strasse 2, 53127 Bonn, Germany

J J Jorzik, H Sieber, S Dithmar, Department of Ophthalmology, University of Heidelberg, INF 400, 69120 Heidelberg, Germany J Dolar-Szczasny, First Eye Hospital of Medical University of Lublin, Chmielna 1, 20-079 Lublin, Poland

C Keilhaver, Department of Ophthalmology, University of Wuerzburg, Josef-Schneider-Strasse 11, 97080 Wuerzburg, Germany

A W A Weinberger, Department of Ophthalmology, University of Aachen, Pauwelsstrasse 30, 52074 Aachen, Germany

D Pauleikhoff, Department of Ophthalmology, St Franziskus Hospital, Domagkstrasse 15, 48149 Muenster, Germany

U Mansmann, Institute for Medical Biometrics and Epidemiology, University of Munich LMU, Marchioninistrasse 15, 81377 Munich, Germany

S Wolf, Department of Ophthalmology, University of Bern Inselspital, 3010 Bern, Switzerland

Supported by Deutsche Forschungsgemeinschaft DFG Ho 1926/2-1, 1926/2-2 DFG Research Priority Program AMD SPP 1088.

\section{APPENDIX}

Centres and members participating in the Fundus Autofluorescence in Age-Related Macular Degeneration (FAM) Study: Department of Ophthalmology, University of Bonn, Germany: investigators: Almut Bindewald, HansMartin Helb, Felix Roth, Steffen Schmitz-Valckenberg, Johannes N Witten, Frank G Holz study nurse: Martina Hofmann; Department of Ophthalmology, University of Heidelberg, Germany: investigators: Jork J Jorzik, Daniel W Miller, Helena Sieber, Stefan Dithmar study nurse: Maria Herrmann Institute for Medical Statistics and Biometry, University of Heidelberg, Germany: Jens Dreyhaupt, Manuela Herzig, Maria Pritsch, Anja Schuldt; Ulrich Mansmann Coordination Center for Clinical Trials, University of Heidelberg: Marcus Schwabedissen, Kristina Unnebrink; Department of Ophthalmology, University of Aachen, Germany: investigator: Andreas Weinberger; Department of Ophthalmology, University of Leipzig, Germany: investigators: Wilma Einbock, Andreas Moessner, Ute Schnurrbusch-Wolf, Henrike Wustemeyer, Sebastian Wolf; 
Department of Ophthalmology, University of Wuerzburg, Germany: investigators: Juliane Mlynski, Claudia Keilhauer; St Franziskus Hospital Muenster, Germany: investigators: Georg Spital, Astrid Meister, Daniel Pauleikhoff.

\section{REFERENCES}

1 Klein R, Klein BE, Jensen SC, et al. The five-year incidence and progression of age-related maculopathy: the Beaver Dam Eye Study. Ophthalmology 1997; 104:7-21.

2 Sunness JS. The natural history of geographic atrophy, the advanced atrophic form of age-related macular degeneration. Mol Vis 1999;5:25-9.

3 Ferris FL 3rd, Fine SL, Hyman L. ge-related macular degeneration and blindness due to neovascular maculopathy. Arch Ophthalmol 1984;102:1640-2.

4 Hyman LG, Lilienfeld AM, Ferris FL 3rd, et al. enile macular degeneration: a case-control study. Am J Epidemiol 1983;118:213-27.

5 Von Rückmann A, Fitzke FW, Bird AC. Distribution of fundus autofluorescence with a scanning laser ophthalmoscope. Br J Ophthalmol 1995;79:407-12.

6 Bellmann C, Holz FG, Schapp O, et al. Topographie der Fundusautofluoreszenz mit einem neuen konfokalen Laser-ScanningOphthalmoskop. Ophthalmologe 1997;94:385-91.

7 Holz FG, Bellmann C, Margaritidis M, et al. Patterns of increased in vivo fundus autofluorescence in the junctional zone of geographic atrophy of the retinal pigment epithelium associated with age-related macular degeneration. Graefes Arch Clin Exp Ophthalmol 1999;237:145-52.

8 Bindewald A, Jorzik JJ, Loesch A, et al. Visualisation of retinal pigment epithelial (RPE) cells in vivo using digital high resolution confocal scanning laser ophthalmoscopy. Am J Ophthalmol 2004;137:556-8.

9 Delori FC, Fleckner MR, Goger DG, et al. Autofluorescence distribution associated with drusen in age-related macular degeneration. Invest Ophthalmol Vis Sci 2000;41:496-504.

10 Delori FC, Dorey CK, Staurenghi G, et al. In vivo fluorescence of the ocular fundus exhibits retinal pigment epithelium lipofuscin characteristics. Invest Ophthalmol Vis Sci 1995;36:718-29.

11 Wing GL, Blanchard GC, Weiter JJ. The topography and age relationship of lipofuscin concentration in the retinal pigment epithelium. Invest Ophthalmol Vis Sci 1978;17:601-7.

12 Weiter JJ, Delori FC, Wing GL, et al. Retinal pigment epithelial lipofuscin and melanin and choroidal melanin in human eyes. Invest Ophthalmol Vis Sci 1986;27:145-52.

13 Dorey CK, Wu G, Ebenstein D, et al. Cell loss in the aging retina. Relationship to lipofuscin accumulation and macular degeneration. Invest Ophthalmol Vis Sci 1989;30:1691-9.

14 Von Rückmann AV, Fitzke FW, Bird AC. Fundus autofluorescence in agerelated macular disease imaged with a laser scanning ophthalmoscope. Invest Ophthalmol Vis Sci 1997;38:478-86.

15 Holz FG, Bellmann C, Staudt S, et al. Fundus autofluorescence and development of geographic atrophy in age-related macular degeneration. Invest Ophthalmol Vis Sci 2001;42:1051-6.

16 Von Rückmann A, Fitzke FW, Bird AC. Distribution of pigment epithelium autofluorescence in retinal disease state recorded in vivo and its change over time. Graefes Arch Clin Exp Ophthalmol 1999;237:1-9.

17 Spaide RF. Fundus autofluorescence and age-related macular degeneration. Ophthalmology 2003;110:392-9.
18 Elkin PL. Primer on medical genomics part V: bioinformatics. Mayo Clin Proc 2003;78:57-64.

19 Gerlai R. Phenomics: fiction or the future? Trends Neurosci 2002;25:506-9.

20 Delori FC, Goger DG, Dorey CK. Age-related accumulation and spatial distribution of lipofuscin in RPE of normal subjects. Invest Ophthalmol Vis Sci 2001;42:1855-66.

21 Feeney-Burns L, Hilderbrand ES, Eldridge S. Aging human RPE: morphometric analysis of macular, equatorial, and peripheral cells. Invest Ophthalmol Vis Sci 1984;25:195-200.

22 Eldred GE, Lasky MR. Retinal age-pigments generated by self-assembling lysosomotropic detergents. Nature 1993;361:724-6.

23 Holz FG, Schutt F, Kopitz J, et al. Inhibition of lysosomal degradative functions in RPE cells by a retinoid component of lipofuscin. Invest Ophthalmol Vis Sci 1999;40:737-43

24 Schutt F, Davies S, Kopitz J, et al. Photodamage to human RPE cells by A2-E, a retinoid component of lipofuscin. Invest Ophthalmol Vis Sci 2000:41:2303-8.

25 Sparrow JR, Nakanishi K, Parish CA. The lipofuscin fluorophore A2E mediates blue light-induced damage to retinal pigmented epithelial cells. Invest Ophthalmol Vis Sci 2000;41:1981-9.

26 Bergmann M, Schütt F, Holz FG, et al. Inhibition of the ATP-driven proton pump in RPE lysosomes by the major lipofuscin fluorophore A2-E may contribute to the pathogenesis of age-related macular degeneration. FASEB J 2004; 18:562-4.

27 Schutt F, Ueberle B, Schnolzer M, et al. Proteome analysis of lipofuscin in human retinal pigment epithelial cells. FEBS Lett 2002;528:217-21.

28 Mata NL, Tzekov RT, Liu X, et al. Delayed dark-adaptation and lipofuscin accumulation in abcr +/- mice: Implications for involvement of $A B C R$ in agerelated macular degeneration 2001:42:1685-90.

29 Ambati J, Anand A, Fernandez S, et al. An animal model of age-related macular degeneration in senescent $\mathrm{Ccl}$-2- or $\mathrm{Ccr}$-2-deficient mice. Nat Med 2003;9:1390-7.

30 Scholl HPN, Bellmann C, Luong V, et al. Photopic and scotopic fine matrix mapping of retinal areas of increased fundus autofluorescence in patients with age-related maculopathy. Invest Ophthalmol Vis Sci 2004; 45:574-83

31 Schmitz-Valckenberg S, Bültmann S, Bindewald A, et al. Fundus autofluorescence and fundus perimetry in the junctional zone of geographic atrophy in patients with age-related macular degeneration. Invest Ophthalmol Vis Sci 2005;45:4470-6.

32 Schmitz-Valckenberg S, Jorzik JJ, et al. FAM Study Group. Analysis of digital scanning laser ophthalmoscopy fundus autofluorescence images of geographic atrophy in advanced age-related macular degeneration. Graefes Arch Clin Exp Ophthalmol 2002;240:73-8.

33 Bellmann C, Jorzik J, Spital G, et al. Symmetry of bilateral lesions in geographic atrophy in patients with age-related macular degeneration. Arch Ophthalmol 2002;120:579-84.

34 Maguire P, Vine AK. Geographic atrophy of the retinal pigment epithelium. Am J Ophthalmol 1986;102:621-5.

35 Sarks JP, Sarks SH, Killingsworth MC. Evolution of geographic atrophy of the retinal pigment epithelium. Eye 1988;2:552-77.

36 Schatz H, McDonald HR. Atrophic macular degeneration. Rate of spread of geographic atrophy and visual loss. Ophthalmology 1989;96:1541-51.

37 Sunness JS, Gonzalez-Baron J, Applegate CA, et al. Enlargement of atrophy and visual acuity loss in the geographic atrophy form of age-related macular degeneration. Ophthalmology 1999;106:1768-79. 